\title{
New Approved First-line Combining Therapy of NSCLC in 2020
}

\author{
Nianwu Wang ${ }^{1}$, Wei Wang ${ }^{3}$, Wenli Mao ${ }^{1}$, Kefeng Wang ${ }^{1}$, Haozhe Zhang ${ }^{1}$, Qiang Ma ${ }^{1}$, Nazuke Kuerbantayi ${ }^{1}$, Yukun \\ Wang*1,2 \\ ${ }^{1}$ School of Medicine, Southern University of Science and Technology, Shenzhen, Guangdong 518055, China \\ ${ }^{2}$ Southern University of Science and Technology Hospital, Shenzhen, Guangdong 518055, China \\ ${ }^{3}$ Department of Pharmaceutics and Pharmacy Administration, School of Pharmacy, Air Force Medical University, Xi'an, Shanxi \\ 710032, China.
}

\begin{abstract}
In the past decades, lung cancer is considered one of the lethal cancers all across the world due to its evidenced greatest mortality and morbidity. Currently, the main clinical treatment methods for lung cancer involve surgery, chemotherapy, radiotherapy, anti-angiogenesis inhibitors and tyrosine kinase inhibitors. In the field of anticancer drugs, a variety of new anticancer drugs have been emerging in recent years. They include chemotherapy drugs with new delivery modes, targeted drugs for tyrosine kinase inhibitors, angiogenesis inhibitors, and immunotherapy drugs that have remained hot. But because cancer cells have complex escape mechanisms, the effectiveness of these single-drug treatments has been disappointing. In this case, a number of new combination therapies have emerged and achieved relatively good results. According to the 2020 third edition of the NCCN guidelines, four multi-therapy regiments (Erlotinib plus ramucirumab, Erlotinib plus bevacizumab, Carboplatin plus albumin-bound paclitaxel plus atezolizumab and Nivolumab plus ipilimumab) have passed clinical trials, adding first-line treatments. In this review, the research provided an overview of these four new approved combining treatment strategies in addition to predicting future drug development directions.
\end{abstract}

\section{Introduction}

In the past decades, lung cancer is considered one of the lethal cancers all across the world due to its evidenced greatest mortality and morbidity. Every year across the globe, roughly 1.8 million people become victim to this deadly disease with a fatality rate of 1.6 million deaths annually.[1] A patient suffering from lung cancer can only live up to 5 years with the chances of beating cancer is not more than only $20 \%$.[1] This low survival chances are contributed to the dangerous side effects accrued from the treatment procedures, the several ways through which lung cancer tissues manifest itself and the complexities involved in the compositions of genes, molecules and cells after its onset. There are basically two categories of lung cancer classified based on the biological characteristics and the mode of clinical treatment, these are non-small cell lung carcinoma (NSCLC) and small cell lung carcinoma (SCLC).

The major type $85 \%$ of the lung cancer cases are of the NSCLC type, (85\%) making it the major type and it is further classified into lung adenocarcinoma (LUAD) and lung squamous cell carcinoma (LUSC).[2] Majority of lung cancer cases are the LUAD type, with a prevalence of $40 \%$. [3] Currently, the main clinical treatment methods for lung cancer involve surgery, chemotherapy, radiotherapy, anti-angiogenesis inhibitors and tyrosine kinase inhibitors.[2] According to the 2020 third edition of the NCCN guidelines, four multi-therapy regiments (Erlotinib plus ramucirumab, Erlotinib plus bevacizumab, Carboplatin plus albumin-bound paclitaxel plus atezolizumab and Nivolumab plus ipilimumab) have passed clinical trials, adding first-line treatments. In this review, the research provided an overview of these four new approved combining treatment strategies in addition to predicting future drug development directions.

\section{Pharmaceutical Achievement for Non- Small Cell Lung Cancer}

\subsection{Chemotherapies}

The most effective treatment option used for several decades for cancers has been chemotherapy and it is still significant in the fight against cancer. Among chemotherapy drugs, cisplatin, an early drug, and platinum drugs based on cisplatin, such as carboplatin and nedaplatin, are more common. Their main mechanism is to trigger DNA cross-linking in cancer cells, preventing them from proliferating indefinitely. In the subsequent drug development, more effective and safer chemotherapeutic drugs have emerged, such as Gemcitabine, Vinorelbine, Paclitaxel, and Pemetrexed.

\footnotetext{
*Corresponding author e-mail: wangyk@sustech.edu.cn
} 


\subsection{Targeted therapies}

However, due to the side effects and poor specificity of traditional chemotherapy drugs, chemotherapy did not achieve satisfactory results for doctors and patients. So, people gradually shifted their attention to targeted drug therapies which have high specificity and safety. Targeted drugs are developed for cancer gene, they are able to identify tumor cells by their unique genetic characteristic and specifically control cell growth and proliferation of tumor cells of signaling pathways, thus kill tumor cells, and prevent its proliferation. Currently, the most widely utilized drugs to treat non-small cell lung cancers are focused on EFGR (mutation frequency approximately $27 \%$ in LUAD, 9\% in LUSC) and ALK (mutation frequency approximately $8 \%$ in LUAD), which are two of mostly activating mutate targets.[2] According to National Comprehensive Cancer Network (NCCN) Guideline, between 2012 and 2019, a number of targeted drugs, such as Afatinib, Erlotinib and Gefitinib for EGFR and Alectinib, Brigatinib and Critinib for ALK, were gradually added to first-line therapies.

\subsection{An Immunological Perspective}

Despite this, the development of anti-cancer drugs has not stopped. As immunological research has increased in the medical field, scientists have tried to assist cancer treatment from an immunological perspective. Therefore, Programmed Death receptor 1, commonly referred to as PD-1, alongside PD-L1 immunotherapy drugs have become hot spots in recent years. As an important immunosuppressive molecule, PD-1 is a member of the CD28 superfamily.[4] Treatment options such as survival of organs during transplantation, fighting tumor and infections and drugs used in fighting autoimmune illness has massively depended on immune regulation targeting PD-1. Its ligand Programmed Cell death-ligand 1 (PD-L1) can also be used as a target, and corresponding antibodies can also play the same role.[5] PD-L1 is Programmed Cell death-ligand 1. It is a transmembrane protein of $40 \mathrm{kDa}$.[6] Whenever the spleen and the lymph nodes have accumulated foreign antigens, there is counterattack response to this accumulation since these new antigens stimulates antigen-specific $\mathrm{T}$ cells to spread. However, this $\mathrm{T}$ cells proliferation can be inhibited by using a combination of PD-L1 and PD-1, which activates inhibitory signals.[7] These two inhibitor drugs used in immunotherapy, that is PD-L1 and PD-1 activates the T cells reactivation and prevents them from reaching their target, hence enabling the immune system to detect the presence of cancerous cells, consequently destroying them.[2] The advantage Because of the drugs is that they have less direct cytotoxicity, and it's safer than the previous two generations.

However, the efficacy of single drug treatments for these drugs still fails to meet overall survival rates, and waiting for a more effective new drug to come to market is costly and time-consuming. Therefore, in the first-line clinical treatment, multi-drug combination therapy has become the trend of anticancer treatment in recent years. According to the 2020 third edition of the NCCN guidelines, four multi-therapy regiments (Erlotinib plus ramucirumab, Erlotinib plus bevacizumab, Carboplatin plus albumin-bound paclitaxel plus atezolizumab and Nivolumab plus ipilimumab) have passed clinical trials, adding first-line treatments.

\section{Combining Therapy of Angiogenesis Inhibitors and Erlotinib}

The efficacy of single drug treatments for lung cancer still fails to meet overall survival rates, and it is costly and time-consuming to waiting for a more effective new drug to come to market. Therefore, in the first-line clinical treatment, multi-drug combination therapy has become the new trend of anticancer treatment in recent years. According to the 2020 third edition of the NCCN guidelines, four multi-therapy regiments (Erlotinib plus ramucirumab, Erlotinib plus bevacizumab, Carboplatin plus albumin-bound paclitaxel plus atezolizumab and Nivolumab plus ipilimumab) have passed clinical trials, adding first-line treatments.

\subsection{Erlotinib}

Erlotinib is used in non-small cell lung cancer as a drug to kill the cancer cells. It specifically impairs the functions of epidermal growth factor receptor (EGFR) through a reverse action of obstructing the EGFR from enabling tyrosine kinase (TK) to do more damage.

3.1.1 Profile of the pathway of EGFR. ErbB cell membrane receptors are the family from which epidermal growth factor receptor (EGFR) arises.[8] ErbB family is responsible for ensuring that the cells differentiate, grow and thrive.[9] Being a transmembrane glycoprotein, EGFR is endowed with extracellular domain whose roles are recognition of particular ligands and then attachment to these ligands. Cell membrane has receptors which interact with the stimulation of the hydrophobic transmembrane domain of EGFR, and lastly the activities of TK enzymes are controlled by its intracellular domain. Heterodimerization or homodimerization of the EGFR only takes place after successful attachment of extracellular domain with the ligands.[9] The important residues of tyrosine located in the cytoplasmic materials undergo autophosphorylation, a process which is stimulated by the TK enzymes already activated during dimerization. Several cellular-docking proteins use these residues from tyrosine as their spots for binding, resulting into a series of downstream signaling which influences the transcription of genes.[8] EGFR has been observed to trigger three downstream signaling pathways and these pathways include Jak2/STAT3, Phosphoinositide-3 kinase (PI3K)/Akt and lastly, the Ras/Raf protein kinase activated by mitogen. Several processes are activated once these signaling pathways have been created. Some of them include preventing the spread of cells, metastasis, invasion, apoptosis prevention and inhibition of angiogenesis. Several types of epithelial tumors are able to facilitate the expression of EFGR.[8] Non-small cell lung cancer 
(NSCLC) is a perfect example of epithelial tumor that is able to manifest EFGR in large quantities through its receptors and ligands. This EFGR expression in NSCLC ranges from 40 to $80 \%$.

3.1.2 Situation of Erlotinib. Erlotinib is therefore designed specifically to inhibit the functions of EGFR extracellular domain. These ranges from preventing attachment of ligands to inhibiting the activation of receptors. Erlotinib also hinders the tyrosine kinase from carrying out activities such as activating the downstream pathways, and inhibiting autophosphorylation of the EGFR.[8] All these processes are aimed at ensuring the cancer cells are effectively destroyed, and anti-cancer inhibitory processes such as metastasis and angiogenesis are prevented. In November, 2004, Food and Drug Administration (FDA) of the United States approved the use of Erlotinib. Erlotinib was first used in 2020 to treat victims of EGFR mutation and metastatic NSCLC, but while supplemented with angiogenesis inhibitors according to the NCCN guideline.

\subsection{Angiogenesis Inhibitors}

The representative drugs used to inhibit angiogenesis in humans by specifically hindering the vascular endothelial growth factor receptors (VEGF) from functioning are Ramucirumab and bevacizumab.[10] Blood vessels carry nutrients and oxygen to tumor cells, which are needed for tumor survival and metastasis to other parts of the body. Metastasis and the growth of tumor are majorly improved by neovascular angiogenesis. Coming from the glycoprotein family, VEGFs therefore have the massive function of activating angiogenesis of the lymph, overall angiogenesis through boosting the growth of the vascular system.[11]

3.2.1 Introduction of VEGF. There are five distinct ligands of VGEFs, which attach themselves to three different TK receptors. These three TK receptors have the following functions: development of hematopoietic cell is controlled by VEGFR-1[12], while the development of endothelial cells in the lymphatic and vascular systems are controlled by VEGFR-3 and VEGFR-2 respectively.[13] After dimerization and attachment of ligands, there are distinct functions of TK assigned to every receptor which are activated. Signaling response from the cytoplasm relies heavily on these TK activities for their signal transmission. A specific protein referred to as VEGF-A is responsible for driving endothelial angiogenesis and its pathological and physiological impacts are mainly mediated by the VEGFR-2. VEGF-A therefore regulates how the endothelial cells spread, survive, migrate and lastly, their permeability. Since VEGFR-2 controls the major pathological impacts of endothelial cells, cancer treatment options should go for its blockade, in order to reduce the growth of tumor and hinder angiogenesis, and from this suggestion, most inhibitors of angiogenesis target the inhibition of VEGFRs in humans.[14]
3.2.2 Situation of Angiogenesis Inhibitors. First line treatment of adenocarcinoma patients was first found to be possible when Bevacizumab is used. The use of this monoclonal antibody to fight the accumulation of VEGFs was authorized after carrying out two successful trials in their third phase. Not only does Bevacizumab identifies and attack VEGFR-2 and VEGFR-1 but it also negates the functions of their associated receptors through biological neutralization.[10] Another monoclinal antibody used specifically against VEGFR-2 in humans is Ramucirumab. It has high attraction to the extracellular domain of the VEGFR-2 hence inhibiting the attachment of ligands.[15] FDA of the United States first authorized the use of ramucirumab in 2014.[16] Its use was allowed after firstline chemotherapy failure among victims of gastroesophageal junction (GEJ) carcinoma and gastric cancer, for both metastatic and advanced cases.[16]After successful early trials, the use of bevacizumab and ramucirumab alongside other chemotherapy drugs was approved for the first-line treatment of NSCLC.

\subsection{Erlotinib Plus Ramucirumab \& Erlotinib Plus Bevacizumab}

The use of gefitinib and erlotinib as the first-generation TKIs drugs during the first therapy for cancer in advanced stages only results into median PFS of 12 months[17-20], even though there are lasting responses. Afatinib, dacomitinib and osimertinib are some of the second and third generation drugs, and they record a mean PFS of $11.0[21-23], 14.7[17]$ and 18.9[24] months respectively. Clinical trials, palliative care and chemotherapy can be used when the first to third generation drugs have been drained. The treatment of EFGR mutations by immune checkpoint inhibitors is still under research since they have not performed effectively. Therefore, focus should be put in developing optimal strategies that inhibits the growth of tumor and prolongs remission especially those focused on EFGR and TKs. Dual blockade strategy has been approved through clinical and preclinical evidence to counter the VEGF and EGFR pathways.

In 2020, NCCN added the combining therapy of erlotinib plus ramucirumab in first-line treatment as an "other recommended" treatment option of category $2 \mathrm{~A}$ and erlotinib plus bevacizumab as a "useful in certain circumstances" treatment option of a category 2B for metastatic NSCLC based on two clinical trials.

The first trial approved the combining therapy of erlotinib plus ramucirumab. This double-blind trial on its third phase was done across the world in 100 health facilities spread on 13 nations.[25] Age limit for participants was 18 and above, but Taiwan and Japan enrolled patients above 20 years during their enrollment time. The NSCLC patients enrolled were in stage IV, and were suffering from either exon 21 substitution (Leu858Arg) or exon 19 deletion (ex19del), EGFR mutations. The two treatment options randomly allocated using the 1:1 ratio was: First option was $150 \mathrm{mg} /$ day of oral erlotinib plus $10 \mathrm{mg} / \mathrm{kg}$ of intravenous ramucirumab and the other option was administering matching placebo every fortnight alongside $150 \mathrm{mg} /$ day of oral erlotinib. 
449 participants joined he study from 28 January, 2016 to 1 February 2018 and 225 were randomly allocated erlotinib plus placebo option and 224 allocated the erlotinib and ramucirumab alternative. 20.7 months was set as the median period for follow-up. The study revealed that treatment option that involved ramucirumab and erlotinib had a longer median PFS of 19.4 months while the second alternative of erlotinib and placebo only yielded median PFS of 12.4 months with a 0.59 stratified hazard ratio. The adverse effects of grade 3 to 4 observed during this study were 121 for the group that used placebo and erlotinib, that is $54 \%$ and the ramucirumab and erlotinib group recorded 159 cases, $72 \%$. Grade 3 Hypertension was the major grade 3 to 4 adverse effects, at 52 cases $(24 \%)$ followed by 33 cases of dermatitis acneiform for the group with ramucirumab and erlotinib. For the placebo and erlotinib group, the major grade 3-4 adverse effects were the 20 cases of dermatitis acneiform followed by the 17 cases of elevated alanine aminotransferase. Serious treatment related adverse events stood at 47 cases $(21 \%)$ for the placebo and erlotinib group and at 65 cases, that is $29 \%$ for the ramucirumab and erlotinib group. Seven cases of pneumonia and four cases of either pneumothorax and cellulitis were the major serious adverse effects regardless of the grade for the ramucirumab and erlotinib group while the top serious adverse effects for the placebo and erlotinib group without considering grade were the four cases of pyrexia and three cases of pneumothorax. There was only one fatality for the ramucirumab and erlotinib group, which was caused by while performing procedure for thoracic drainage leading to hemothorax. The study revealed that combining erlotinib with ramucirumab results into increased treatment related adverse events compared to using erlotinib and placebo but this disparity was only a margin of one to 2 grade.[25] Besides, when supportive care and dose reductions are applied, these adverse effects can be managed efficiently. For the firstline NSCLC patients suffering from metastatic EGFR mutations, adopting treatment combination of erlotinib and ramucirumab yields longer median PFS than treatment combination of erlotinib and placebo. One crucial factor in this study was ensuring safety for the NSCLC patients and also for the single treatment compounds by creating safety profiles. NSCLC patients with EGFR mutations can safely apply this RELAY regime for their first-line treatment.

This involved analyzing an open-labeled and randomized trial in its third phase through predetermined interim approach. The trial involved NSCLC patients at either stage IV or IIIB and non-squamous type whether confirmed histologically, cytologically or recurrent but exhibits activation of the genomic aberrations of EGFR.[26] The trial was done in Japan across 69 health facilities and the age limit for patients was set at 20 and above, who had not undergone any chemotherapy treatments, and their ECOG performance statuses for their organs were not more than 2 . The first treatment option was the addition of $15 \mathrm{mg} / \mathrm{kg}$ of intravenous bevacizumab administered once after three weeks, to $150 \mathrm{mg} /$ day of erlotinib. Second treatment was a daily monotherapy of taking $150 \mathrm{mg}$ of erlotinib orally. The population was stratified in accordance with the subtype of their EGFR mutation, sex, cancer stage and smoking status while using minimization approach to randomly allocate them treatment options. For the results observed from 3 June, 2015 to 31 August, 2016, of the 228 patients enrolled, 114 were allocated the erlotinib and bevacizumab option while the remaining 114 assigned only erlotinib. For efficacy evaluation, each group provided 112 participants, but the combination group and erlotinib-alone group provided 112 and 114 patients for safety evaluation respectively. 12.4 months was the set median period for follow-up. The interim analysis revealed that combination therapy group accrued a median PFS of 16.9 months compared to the monotherapy group that only yielded 13.3 months median PFS. While the combination therapy group recorded 98 cases, that is $88 \%$, of either grade 3 or more serious adverse events, the monotherapy group only recorded 53 cases for the same events. Two cases of grade 4 neutropenia and one case of grade 4 hepatic dysfunction were the major serious adverse events reported in the combination therapy group. For the monotherapy group, the top serious adverse event was a lone case of hepatic dysfunction. Luckily, no fatalities were caused by these adverse events. This study revealed that combination of erlotinib with bevacizumab results into longer median PFS for NSCLC patients with EGFR mutations compared to when they are treated with erlotinib alone.[26] For these reasons, combination therapy involving erlotinib and bevacizumab has a massive capacity to be adopted as standard treatment option for NSCLC patients with EGFR mutations especially if life quality improves and survival chances increased.

\section{Combining Therapy of Chemotherapeutic Drugs and Immune Checkpoint Inhibitors}

\subsection{Platinum-based Chemo Therapy}

Platinum based chemotherapy currently includes three generations. The representative drug of the first generation is cisplatin, which belongs to a broad spectrum of antitumor drugs. Cisplatin has no obvious selectivity against tumor sites, with obvious side effects and good anti-tumor effects. The toxicological effect of cisplatin is the main mechanism of platinum chemotherapeutic drugs.[27, 28] In the second generation, the representatives of drugs are carboplatin and nedaplatin. This generation of drugs is more water-soluble and has fewer side effects. Due to the increased water-solubility, the toxicity to the kidney is reduced. The third-generation drugs include oxaliplatin and loplatin, which are mainly suitable for the treatment of resistance to cisplatin, the first-generation anticancer drug. Oxaliplatin has a wider range of clinical application, but oxaliplatin is neurotoxic and has obvious symptoms of thrombocytopenia caused by loplatin. In the $2020 \mathrm{NCCN}$ guideline, combination therapy of carboplatin plus albumin-bound paclitaxel plus atezolizumab is approved for first-line NSCLC treatment. 
4.1.1 Action of Platinum-based Chemotherapy. Patients suffering from ovarian cancer have always vouched for platinum-based second-generation drug, Carboplatin and there is huge possibility that squamous cell carcinomas, which is a type of SCLC, affecting the neck and hand, alongside seminomas can be treated by Carboplatin. General analysis show that it has the same range of qualitative activities as cisplatin. The main mechanism of antitumor activity of the platinum complexes is thought to be related to their ability to react with nucleophilic sites on DNA.[27] Cisplatin undergoes hydrolysis (loss $\mathrm{Cl}$ in cisplatin and dicarboxylate group in carboplatin) in solution and the monoaquated species which is formed reacts readily with DNA, forming intrastrand and DNA-protein crosslinks. Since, cisplatin has great nucleophilicity for the N7 position in the adenine and guanine, studies of complete DNA shows that this position is its preferential attachment site.[29] The development of intrastrand N-7 adduct with dual functions at both $\mathrm{d}(\mathrm{ApG})$ and $\mathrm{d}(\mathrm{GpG})$, is linked with the role played by cisplatin in fighting the cultured neoplastic cells through release of cytotoxins, and it has close correlation with intrastrand cross-links of the platinum DNA.[30-32] Similar behavior is evidenced in both cisplatin and Carboplatin. Reaction of cells' nucleophilic sites on DNA with carboplatin stimulates the formation of these crosslinks: DNA-protein, interstrand and intrastrand crosslinks. [31, 32] However, cisplatin stimulates the formation of these cross-links at higher rate than carboplatin. The dicarboxylate group in carboplatin is much more stable than the chloride groups of cisplatin, which results in carboplatin being less hydrolytic than cisplatin. In a demonstration to prove that both cisplatin and carboplatin stimulates the formation of platinum-DNA adducts, pUC18 plasmid DNA was used by Hongo and coworkers.[33] This demonstration revealed that for the plasmid DNA to undergo the same extent of confrontational modification, the incubation period of carboplatin was 7.5 times higher than that of cisplatin and carboplatin needed 10 times greater concentration. Since carboplatin exerts a milder effect than cisplatin, it has a higher safety profile.

\subsection{Albumin-bound Paclitaxel}

\subsubsection{Action of Paclitaxel-based Chemotherapy.} Paclitaxel is an organic compound which is found in the back of Pacific yew. It is diterpenoid and hydrophobic. Its role as an antineoplastic driver has enabled its successful application in the treatment of several cancers such as NSCLC, ovarian and breast cancers. It influences the stabilization and assembling of microtubules made from tubulin dimers, hence act as an antimicrotubular agent by inhibiting their depolymerization. After making the microtubule stable, they cannot be rearranged in to their normal dynamic patterns, hence impairing processes such as function of mitotic cells and the crucial interphase which depends on this microtubule reorganization. Instead of the cell undergoing mitosis to create more cells, the abnormal patterns of microtubule and their increased stability which is caused by paclitaxel results into consequential cell deaths.[34] Ethanol and polyethylene castor oil are among the organic reagents that were used in the earlier years to clinically dissolve paclitaxel since it is hydrophobic. The pharmacological and biological characteristics of these organic reagents are major contributors to toxicities.

Albumin-bound delivery technology enter human's vision. Based on the three characteristics of this substance, albumin is judged to be a carrier of paclitaxel: First reason, is that albumin forms reversible non-covalent bonds with hydrophobic compounds, which is only required for transportation, but once it reaches the cell surface it releases the molecules easily[35]; Secondly, once albumin is attached to the receptors, it stimulates the movement of the constituents of plasma, whether bound or unbound with albumin from the endothelial cell to the space between the cells.[36-42] Thirdly, the osteonectin secreted by albumin favors accumulation of albumin within the tumors hence resulting in whatever molecule the albumin was carrying to also accumulate and in this case, it is paclitaxel.[43-46] Based on this conjecture the nab (130-nanometer albumin-bound) Technology was successfully used in the delivery of paclitaxel. nab Technology, applies the nature of albumin to devise a faster transport of drugs to the malignant tissues, and this system is responsive to the biology of human beings. The non-covalent bonds that albumin forms with these molecules, do not result to any modification to their properties. ABI-007(Abraxane $\AA$, nab-paclitaxel) is the initial marketable product formed using nab Technology and it does not need any organic solvent.[47] The combination of albumin and paclitaxel in this product is referred to as nab-paclitaxel, with a concentration of 130 $\mathrm{nm}$. It contains nanoparticles of lyophilized paclitaxel cakes, which are further divided into human albumin of $900 \mathrm{mg}$ and paclitaxel of $100 \mathrm{mg}$ for every vial of $50 \mathrm{ml}$. $20 \mathrm{ml}$ of sodium chloride at $0.9 \%$ is injected to reconstitute a suspension of $1 \mathrm{ml}$ volume which only have paclitaxel nanoparticles weighing $5 \mathrm{mg}$. In vitro studies show that when nab particles are dissolved in solution, they diffuse into paclitaxel-bound albumin molecules; the same reaction that occurs immediately after being injected in the blood steam.

\subsection{Immune Checkpoint Inhibitor Antibodies}

The research work within the past recent decades, has been met with great zeal as researchers and scientists explore special ways of fighting cancers such as use of cytokine therapies, vaccines for cancers and monoclonal antibodies as was of boosting the immune system. Blocking of the immune checkpoints (ICPs) of stimulate activities that inhibits tumor development is among the appealing procedures.[48] This ICPs blockade seems to have a huge potential in the field of cancer research since it involves modifications to the microenvironment of the tumor. Presence of inhibitory molecules, hence no costimulatory molecules hinders the process through which $\mathrm{T}$ cells are activated.[49] 


\subsubsection{Action Mechanism of anti-CTLA-4 Antibodies.} T-cell activation is a complex process that requires $>1$ stimulatory signal. TCR binding to MHC provides specificity to T-cell activation, but further costimulatory signals are required. Binding of B7-1 (CD80) or B7-2 (CD86) molecules on the APC (Antigen Presenting Cells) with $\mathrm{CD} 28$ molecules on the $\mathrm{T}$ cell leads to signaling within the T cell.[50] Sufficient levels of CD28:B7-1/2 binding lead to proliferation of $\mathrm{T}$ cells, increased T-cell survival, and differentiation through the production of growth cytokines such as interleukin-2 (IL-2), increased energy metabolism, and upregulation of cell survival genes. CTLA-4 is a CD28 homolog with much higher binding affinity for $\mathrm{B} 7$; however, unlike $\mathrm{CD} 28$, binding of CTLA-4 to B7 does not produce a stimulatory signal.[50] As such, this competitive binding can prevent the costimulatory signal normally provided by CD28:B7 binding. The relative amount of $\mathrm{CD} 28: \mathrm{B} 7$ binding versus CTLA-4: B7 binding determines whether a $T$ cell will undergo activation or anergy. Furthermore, some evidence suggests that CTLA-4 binding to B7 may actually produce inhibitory signals that counteract the stimulatory signals from CD28:B7 and TCR: MHC binding. Proposed mechanisms for such inhibitory signals include direct inhibition at the TCR immune synapse, inhibition of CD28 or it signaling pathway, or increased mobility of T cells leading to decreased ability to interact with APCs. CTLA-4 itself is subject to regulation, particularly by localization within the cell. In resting naive T cells CTLA-4 is located primarily in the intracellular compartment. Stimulatory signals resulting from both TCR and CD28:B7 binding induce upregulation of CTLA- 4 on the cell surface by exocytosis of CTLA-4containing vesicles. This process operates in a graded feedback loop whereby stronger TCR signaling elicits more CTLA-4 translocation to the cell surface. In case of a net negative signal through CTLA-4: B7 binding, full activation of $\mathrm{T}$ cells is prevented by inhibition of IL-2 production and cell cycle progression. CTLA-4 is also involved in other aspects of immune control. Regulatory $\mathrm{T}$ cells (Tregs) control functions of the effector $\mathrm{T}$ cells, and thus are key players in maintaining peripheral tolerance. Unlike effector $\mathrm{T}$ cells, Tregs constitutively express CTLA-4, and this is thought to be important for their suppressive functions. In animal models, genetic CTLA-4 deficiency in Tregs impaired their suppressive functions. One mechanism whereby Tregs are thought to control effector T cells is downregulation of B7 ligands on APCs, leading to reduced CD28 stimulation.

Ipilimumab was the initial ICP inhibitor to undergo clinical investigation. This monoclonal antibody works by preventing the CTLA4 receptors located on T cells from interacting with $\mathrm{B} 7$, hence commonly referred to as antiCTLA4 antibody. The FDA authorized the use of ipilimumab in 2011, especially due to its significance in fighting metastatic melanoma.

4.3.2 Action Mechanism of anti-PD-1 Antibodies. When PD1 interacts with its major ligands such as PDL2 and PDL1, they create another ICP. Some of the several immune cells that exhibit the expression of PD1 include Tregs, activated $\mathrm{T}$ cells, dendritic cells, natural killer cells and monocytes. PD-1 is a member of the B7/CD28 family of costimulatory receptors.[51] It regulates T-cell activation through binding to its ligands, programmed death ligand 1 (PD-L1) and programmed death ligand 2 (PD-L2). Similar to CTLA-4 signaling, PD-1 binding inhibits T-cell proliferation, and interferon-g (IFN-g), tumor necrosis factor-a, and IL-2 production, and reduces T-cell survival.[52] If a T cell experiences coincident TCR and PD-1 binding, PD-1-generated signals prevent phosphorylation of key TCR signaling intermediates, which terminates early TCR signaling and reduces activation of T cells. PD-1 expression is a hallmark of exhausted $\mathrm{T}$ cells that have experienced high levels of stimulation or reduced CD4T-cell help. This state of exhaustion, which occurs during chronic infections and cancer, is characterized by T-cell dysfunction, resulting in suboptimal control of infections and tumors. The attachment of PD1 cells to tumor-infiltrating immune cells (ICs) and tumor cells (TCs) in a region with growing tumor tissue results into TDL1 expressions whose roles are to terminate any activity by the $\mathrm{T}$ cells aimed at fighting the tumor. Not only can the tumor cells stimulate the tumor-infiltrating immune cells to express the PD1 ligands but also allow these TD1 ligands to express on their surface creating a complete barrier to immunosurveillance.[53]

The huge potential that monoclonal antibodies seem to have in inhibiting the PD1 from interacting with its ligands, has attracted massive research in the field of cancer therapeutics. Nivolumab, pembrolizumab and ipilimumab are some of the many PD1-targeted monoclonal antibodies that have been created. Nivolumab has always been the center of research, forcing the FDA to approve it after successful trials in treating metastatic renal cell carcinoma, metastatic melanoma, Hodgkin lymphoma and majorly, NSCLC. PDL1 has been found to be the most crucial ligands of PD1 unlike PDL2. One major function of PDL1 is regulating the lymphocytes. Inhibition of PD-L2 binding leads to enhanced TH2 activity, whereas PD-L1 binding to CD80 has been shown to inhibit $\mathrm{T}$ cell responses. These different biological effects are likely to contribute to differences in activity and toxicity between antibodies directed at PD-1 (preventing binding to both ligands) as opposed to those directed at PD-L1, and therefore have potential therapeutic implications. Therefore, significant antibodies such as avelumab, durvalumab and atezolizumab have been identified to specifically inhibit TDL1.[54]

In 2020, the NCCN guideline approved the combination therapy of ipilimumab plus nivolumab is approved for first-line NSCLC treatment according to a phase 3 clinical trial which also shows their efficacy compared with chemotherapy medication.

\subsection{Nivolumab plus Ipilimumab}

The combination therapy of nivolumab and ipilimumab was approved by the NCCN guideline after a randomized trial on its third phase which involved participants 
suffering from recurrent NSCLC in stage IV, and based on percentage expression of the PD-L1.[55] The eligible participants had not undertaken any earlier chemotherapy, and for this study, overall survival was used as the end point. The experiment was done for two case scenarios; first one was for patients whose expression level of the PD-L1 was at least $1 \%$ and secondly, patients whose expression level of the PD-L1 was not more than $1 \%$. And for both scenarios, randomization ratio of 1:1:1 was used to allocate patients three treatment options: chemotherapy alone, nivolumab alone, and combination therapy of nivolumab and ipilimumab.

For those participants whose expression level of PDL1 stood at Tumor-associated B7-H1 promotes T-cell apoptosis: a potential mechanism of immune evasion. at least $1 \%$, the results indicated that those who adopt combination therapy of nivolumab and ipilimumab recorded a median overall survival duration of 17.1 months, with the chemotherapy group patients recording only 14.9 months of overall survival duration. Their respective percentage rates of survival were $40.0 \%$ and $32.8 \%$ for the 2 -year period. While chemotherapy alone showed only 6.2 months of median response duration, for the combined therapy stood majestically at 23.2 months. The same trend was also observed for the patients whose expression levels of the PD-L1 were at not more than $1 \%$. The combined therapy of nivolumab and ipilimumab still recorded higher median overall survival duration of 17.2 months compared to the 12.2 months recorded by the chemotherapy-only participants. Regardless of the PD-L1 expression level, patients from the combined therapy group recorded a mean overall survival duration of 17.1 months compared to the 13.9 months exhibited among chemotherapy-only patients. Besides, patients from the combined therapy group suffered less treatment-related adverse events than chemotherapy-only patients, $32.8 \%$ versus $36.0 \%$ prevalence.[55] Therefore, this study proved that enhancing first-line treatment of NSCLC by combining nivolumab with ipilimumab makes the mean overall survival duration longer than if only chemotherapy is applied and this is not affected by the expression levels of the PD-L1.

\subsection{Carboplatin plus Albumin-bound Paclitaxel plus Atezolizumab}

The NCCN guideline give the approval for carboplatin plus albumin-bound paclitaxel plus atezolizumab combining therapy. This approval was given based on an open-labeled, multi-centered, randomized study on its third phase spread across Germany, Israel, Belgium, the USA, Spain, Italy, France, and Canada in a total of 131 health facilities.[56] The age limits for participants was 18 and above, with these NSCLC patients required to not have gone any chemotherapy and have an ECOG performance status of not more than one, and their cancer is on its stage IV and it is of the non-squamous type. Randomization through voice-response system was used to allocate treatment options. The first treatment option involved adding $1200 \mathrm{mg}$ of intravenous atezolizumab to be administered after every 21 days alongside chemotherapy involving both carboplatin and nabpaclitaxel. $6 \mathrm{mg} / \mathrm{mL}$ per minute of carboplatin is done after every 21 days while $100 \mathrm{mg} / \mathrm{m}^{2}$ of intravenous nabpaclitaxel is done on a weekly basis. The second option is four to six chemotherapy cycles alone, which are done after every 3 weeks, and then maintenance therapy. The participants were stratified based on expression of PD-L1 tumor, baseline metastasis of the liver and sex. The researchers assessed the PFS to determine the co-primary endpoints while for the ALK and EGFR population, overall survival was the used primary endpoints. Any participant who took more than a single dose of the drugs had to be under safety guidelines.

724 eligible patients were enrolled in the study as from 16 April, 2015 to $13 \mathrm{Feb}, 2017$, and joined the intentionto-treat population but one participant had to be excluded from this population since they died before treatment allocation. There were two categories of intention-to-treat populations: the normal type and the wild type. For the group that combined chemotherapy with atezolizumab, the wild-type population contained 452 of the participants while the normal type population had 483 participants. For the chemotherapy-alone group, the wild-type population contained 228 participants while the normal type population contained 240 participants. The two groups recorded almost similar median follow up, with the chemotherapy-alone group having median follow-up of 19.2 months and the chemotherapy and atezolizumab group having median follow up of 18.5 months for the wild-type population. The medial overall survival increased in both groups for the wild-type population, with the chemotherapy-alone group recording 13.9 months and the chemotherapy and atezolizumab group recording 18.6 months. The mean PFS for the wild-type population stood at 5.5 months and 7.0 months respectively. For neutropenia there were 152 cases from 473 patients, and 65 cases from 232 patients from chemotherapy and atezolizumab group and chemotherapy-alone group respectively, making it the most prevalent grade 3 or worse treatment-related events. For the chemotherapy and atezolizumab group, and chemotherapy-alone group, anemia cases were 138 and 47, cases of decreased neutrophil count were 57 and 19 respectively. The same trend was witnessed for other treatment-related serious adverse events whereby chemotherapy and atezolizumab patients topped with 112 when the chemotherapy group only had 30 cases. The chemotherapy and atezolizumab group suffered 8 fatalities due to adverse events while the chemotherapyonly group reported only one death.[56] Therefore, the use of atezolizumab alongside chemotherapy for the first line treatment of non-squamous NSCLC within stage IV with no EFGR or ALK mutations results into the increase in the mean PFS and the overall survival compared to when only chemotherapy is used. There were no any identified safety issues. This study therefore endorses the adoption of atezolizumab alongside platinum-based chemotherapy since it has proved this combination is beneficial to firstline treatment of NSCLC. 


\section{Conclusion}

In this review, we provide a comprehensive overview of the mechanisms of action and clinical efficacy of each of the four combining therapy strategies that have been added to first-line treatment of metastatic non-small cell lung cancer in 2020. These combinations include the targeted erlotinib plus angiogenic inhibitors, ramucirumab and bevacizumab; platinum-based chemo therapy drug carboplatin plus paclitaxel-based chemo therapy drug albumin-bound paclitaxel and PD-L1 immune checkpoint inhibitor atezolizumab; PD-1 inhibitor nivolumab and anti-CTLA4 antibody ipilimumab. NCCN guidelines for the treatment of non-small cell lung cancer in 2020 compared with previous years, the proportion of multidrug combination therapy was significantly increased. Such multi-drug combination therapy may become an important trend in clinical pharmacy development in the next few years, because the development of a more effective specific anti-cancer drug requires a long-time investment of five to ten years, but multi-drug combination therapy tends to take less time, and multidrug combination therapy often shows significantly better therapeutic effect. For example, erlotinib and ramucirumab were compared with erlotinib and placebo in randomized clinical intervention trials. As a result, treatment option that involved ramucirumab and erlotinib had a longer median PFS of 19.4 months while the second alternative of erlotinib and placebo only yielded median PFS of 12.4 months.[25] The combination was thought to be nearly a half more effective as the single drug, so the search for combination regimens is going to be critical. However, not all anticancer drugs can be used at the same time without any hesitation. Based on the relevant knowledge introduced in this review, we can try to speculate on several basic institutions of combined drug use.

First, the two or more drugs need to have similar action by targeting different targets. If two drugs have the same target, the drug molecules cannot bind to both targets. In this case, multi-drug combination therapy will be difficult to make a difference compared with single-drug therapy, because the newly added drug does not combine the target to perform its corresponding function. Accordingly, if two drugs do not have similar functions but perform their respective functions, there will be no synergy between them to make a significant difference in efficacy. For instance, erlotinib targeting EGFR while angiogenesis inhibitors targeting VEGFR, both EGFR and VEGFR could induce angiogenesis. In this case, their combination resulted in a highly effective synergistic effect on the inhibition of new angiogenesis, resulting in a significant increase in the effectiveness of treatment. Another example is the combination of nivolumab and ipilimumab. The aim of both drugs is to activate $T$ cells to remove cancer cells. Nivolumab targets PD1, while ipilimumab targets CTLA4. They also have similar functions and combine different targets. Both CTLA-4 and PD-1 binding have similar negative effects on T-cell activity; however, the timing of downregulation, the responsible signaling mechanisms, and the anatomic locations of immune inhibition by these 2 immune checkpoints differ.
Unlike CTLA-4, which is confined to T cells, PD-1 is more broadly expressed on activated T cells, B cells, and myeloid cells. While CTLA-4 functions during the priming phase of T-cell activation, $\mathrm{PD}-1$ functions during the effector phase, predominantly within peripheral tissues. When both ICPs are inhibited, T cell activity is significantly liberated as a result of this synergistic effect, producing a potency that cannot be achieved by single drug therapy.

Second, combination or alternating therapy requires a relatively high level of safety. Carboplatin was generally considered less toxic than cisplatin, and Albumin-bound paclitaxel was less toxic than paclitaxel. Therefore, the concurrent use of two chemotherapy drugs can ensure a certain degree of safety. Although the combination of cisplatin and paclitaxel has appeared in many clinical trials over the past few years, the use of both cisplatin and paclitaxel is not recommended for first-line treatment, according to the 2020 NCCN Guidelines for non-small cell lung Cancer treatment. This may be due to the emergence of new fewer toxic drugs which lead to the phase out of highly toxic treatment strategies.

In summary, a variety of new multi-drug combinations for NSCLC are rapidly increasing, providing hope for improved survival in patients with metastatic NSCLC in the short term. But the combination therapy with existing drugs still fails to meet the expectations of non-small cell lung cancer, so new and more effective drugs remain the key to a cancer breakthrough, which is emergency and need further study.

\section{References}

1. Hirsch F R, Scagliotti G V, Mulshine J L, Kwon R, Curran W J, Wu Y-L and Paz-Ares L 2017 Lung cancer: current therapies and new targeted treatments The Lancet 389 299-311

2. Herbst R S, Morgensztern D and Boshoff C 2018 The biology and management of non-small cell lung cancer Nature 553 446-54

3. Denisenko T V, Budkevich I N and Zhivotovsky B 2018 Cell death-based treatment of lung adenocarcinoma Cell Death Dis 9117

4. Kobold S, Grassmann S, Chaloupka M, Lampert C, Wenk S, Kraus F, Rapp M, Düwell P, Zeng Y, Schmollinger J C, Schnurr M, Endres $\mathrm{S}$ and Rothenfußer S 2015 Impact of a New Fusion Receptor on PD-1-Mediated Immunosuppression in Adoptive T Cell Therapy $J$ Natl Cancer Inst 107

5. Lantuejoul S, Damotte D, Hofman V and Adam J 2019 Programmed death ligand 1 immunohistochemistry in non-small cell lung carcinoma Journal of thoracic disease 11 S89-s101

6. Keir M E, Liang S C, Guleria I, Latchman Y E, Qipo A, Albacker L A, Koulmanda M, Freeman G J, Sayegh M H and Sharpe A H 2006 Tissue expression of PD-L1 mediates peripheral T cell tolerance The Journal of experimental medicine 203 883-95 
7. Dong Y, Sun Q and Zhang X 2017 PD-1 and its ligands are important immune checkpoints in cancer Oncotarget 8 2171-86

8. Bareschino M A, Schettino C, Troiani T, Martinelli E, Morgillo F and Ciardiello F 2007 Erlotinib in cancer treatment Ann Oncol 18 Suppl 6 vi35-41

9. Ciardiello F and Tortora G 2001 A novel approach in the treatment of cancer: targeting the epidermal growth factor receptor Clinical cancer research : an official journal of the American Association for Cancer Research 7 2958-70

10. Manzo A, Montanino A, Carillio G, Costanzo R, Sandomenico C, Normanno N, Piccirillo M C, Daniele G, Perrone F, Rocco G and Morabito A 2017 Angiogenesis Inhibitors in NSCLC Int J Mol Sci 18

11. Ferrara N 1999 Molecular and biological properties of vascular endothelial growth factor Journal of molecular medicine (Berlin, Germany) 77 527-43

12. Ferrara N, Gerber H P and LeCouter J 2003 The biology of VEGF and its receptors Nature medicine 9 669-76

13. Robinson D R, Wu Y M and Lin S F 2000 The protein tyrosine kinase family of the human genome Oncogene $195548-57$

14. Hall R D, Le T M, Haggstrom D E and Gentzler R D 2015 Angiogenesis inhibition as a therapeutic strategy in non-small cell lung cancer (NSCLC) Translational lung cancer research 4 515-23

15. Aprile G, Rijavec E, Fontanella C, Rihawi K and Grossi F 2014 Ramucirumab: preclinical research and clinical development Onco Targets Ther 7 1997-2006

16. Poole R M and Vaidya A 2014 Ramucirumab: first global approval Drugs 74 1047-58

17. Wu Y L, Cheng Y, Zhou X, Lee K H, Nakagawa K, Niho S, Tsuji F, Linke R, Rosell R, Corral J, Migliorino M R, Pluzanski A, Sbar E I, Wang T, White J L, Nadanaciva S, Sandin R and Mok T S 2017 Dacomitinib versus gefitinib as first-line treatment for patients with EGFR-mutation-positive non-smallcell lung cancer (ARCHER 1050): a randomised, open-label, phase 3 trial The Lancet. Oncology 18 1454-66

18. Rosell R, Carcereny E, Gervais R, Vergnenegre A, Massuti B, Felip E, Palmero R, Garcia-Gomez R, Pallares C, Sanchez J M, Porta R, Cobo M, Garrido P, Longo F, Moran T, Insa A, De Marinis F, Corre R, Bover I, Illiano A, Dansin E, de Castro J, Milella M, Reguart N, Altavilla G, Jimenez U, Provencio M, Moreno M A, Terrasa J, Muñoz-Langa J, Valdivia J, Isla D, Domine M, Molinier O, Mazieres J, Baize N, Garcia-Campelo R, Robinet G, Rodriguez-Abreu D, Lopez-Vivanco G, Gebbia V, Ferrera-Delgado L, Bombaron P, Bernabe R, Bearz A, Artal A, Cortesi E, Rolfo C, Sanchez-Ronco M, Drozdowskyj A, Queralt C, de Aguirre I, Ramirez J L, Sanchez J J, Molina M A, Taron M and Paz-Ares L 2012 Erlotinib versus standard chemotherapy as first-line treatment for European patients with advanced EGFR mutationpositive non-small-cell lung cancer (EURTAC): a multicentre, open-label, randomised phase 3 trial The Lancet. Oncology 13 239-46

19. Zhou C, Wu Y L, Chen G, Feng J, Liu X Q, Wang C, Zhang S, Wang J, Zhou S, Ren S, Lu S, Zhang L, Hu C, Hu C, Luo Y, Chen L, Ye M, Huang J, Zhi X, Zhang Y, Xiu Q, Ma J, Zhang L and You C 2011 Erlotinib versus chemotherapy as first-line treatment for patients with advanced EGFR mutation-positive non-small-cell lung cancer (OPTIMAL, CTONG0802): a multicentre, open-label, randomised, phase 3 study The Lancet. Oncology 12 735-42

20. Mitsudomi T, Morita S, Yatabe Y, Negoro S, Okamoto I, Tsurutani J, Seto T, Satouchi M, Tada H, Hirashima T, Asami K, Katakami N, Takada M, Yoshioka H, Shibata K, Kudoh S, Shimizu E, Saito H, Toyooka S, Nakagawa K and Fukuoka M 2010 Gefitinib versus cisplatin plus docetaxel in patients with non-small-cell lung cancer harbouring mutations of the epidermal growth factor receptor (WJTOG3405): an open label, randomised phase 3 trial The Lancet Oncology 11 121-8

21. Park K, Tan E H, O'Byrne K, Zhang L, Boyer M, Mok T, Hirsh V, Yang J C, Lee K H, Lu S, Shi Y, Kim S W, Laskin J, Kim D W, Arvis C D, Kölbeck K, Laurie S A, Tsai C M, Shahidi M, Kim M, Massey D, Zazulina V and Paz-Ares L 2016 Afatinib versus gefitinib as first-line treatment of patients with EGFR mutation-positive non-small-cell lung cancer (LUXLung 7): a phase 2B, open-label, randomised controlled trial The Lancet. Oncology 17 577-89

22. Wu Y L, Zhou C, Hu C P, Feng J, Lu S, Huang Y, Li W, Hou M, Shi J H, Lee K Y, Xu C R, Massey D, Kim M, Shi Y and Geater S L 2014 Afatinib versus cisplatin plus gemcitabine for first-line treatment of Asian patients with advanced non-small-cell lung cancer harbouring EGFR mutations (LUX-Lung 6): an open-label, randomised phase 3 trial The Lancet. Oncology 15 213-22

23. Sequist L V, Yang J C, Yamamoto N, O'Byrne K, Hirsh V, Mok T, Geater S L, Orlov S, Tsai C M, Boyer M, Su W C, Bennouna J, Kato T, Gorbunova V, Lee K H, Shah R, Massey D, Zazulina V, Shahidi M and Schuler M 2013 Phase III study of afatinib or cisplatin plus pemetrexed in patients with metastatic lung adenocarcinoma with EGFR mutations $J$ Clin Oncol 31 3327-34

24. Soria J C, Ohe Y, Vansteenkiste J, Reungwetwattana $\mathrm{T}$, Chewaskulyong B, Lee K H, Dechaphunkul A, Imamura F, Nogami N, Kurata T, Okamoto I, Zhou C, Cho B C, Cheng Y, Cho E K, Voon P J, Planchard D, Su W C, Gray J E, Lee S M, Hodge R, Marotti M, Rukazenkov Y and Ramalingam S S 2018 Osimertinib in Untreated EGFR-Mutated Advanced Non-Small-Cell Lung Cancer N Engl J Med 378 11325

25. Nakagawa K, Garon E B, Seto T, Nishio M, Ponce Aix S, Paz-Ares L, Chiu C-H, Park K, Novello S, Nadal E, Imamura F, Yoh K, Shih J-Y, Au K H, Moro-Sibilot D, Enatsu S, Zimmermann A, FrimodtMoller B, Visseren-Grul C, Reck M, Chu Q, Cortot 
A, Pujol J-L, Moro-Sibilot D, Fabre E, Lamour C, Bischoff H, Kollmeier J, Reck M, Kimmich M, Engel-Riedel W, Hammerschmidt S, Schütte W, Syrigos K, Ho J C M, Au K-H, Novello S, Ardizzoni A, Pasello G, Gregorc V, Del Conte A, Galetta D, Takahashi T, Nakagawa K, Nishio M, Yoh K, Seto T, Imamura F, Kumagai T, Hotta K, Goto Y, Hosomi Y, Sakai H, Takiguchi Y, Kim Y H, Kurata T, Yamaguchi H, Daga H, Okamoto I, Satouchi M, Ikeda S, Kasahara K, Atagi S, Azuma K, Kumagai T, Aoe K, Kumagai T, Aoe K, Horio Y, Yamamoto N, Tanaka H, Watanabe S, Nogami N, Ozaki T, Koyama R, Hirashima T, Kaneda H, Tomii K, Fujita Y, Seike M, Nishimura N, Kato T, Ichiki M, Saka H, Hirano K, Nakahara Y, Sugawara S, Park K, Kim S-W, Min Y J, Lee H W, Kang J-H, An H J, Lee K H, Kim J-S, Lee G-W, Lee S Y, Alexandru A, Udrea A A, JuanVidal Ó, Nadal-Alforja E, Gil-Bazo I, Ponce-Aix S, Paz-Ares L, Rubio-Viqueira B, Alonso Garcia M, Felip Font E, Fuentes Pradera J, Coves Sarto J, Lin M-C, Su W-C, Hsia T-C, Chang G-C, Wei Y-F, Chiu C-H, Shih J-Y, Su J, Cicin I, Goksel T, Harputluoglu H, Ozyilkan O, Henning I, Popat S, Hatcher O, Mileham K, Acoba J, Garon E, Jung G, Raj M, Martin W and Dakhil S 2019 Ramucirumab plus erlotinib in patients with untreated, EGFR-mutated, advanced non-small-cell lung cancer (RELAY): a randomised, double-blind, placebo-controlled, phase 3 trial The Lancet Oncology 20 1655-69

26. Saito H, Fukuhara T, Furuya N, Watanabe K, Sugawara S, Iwasawa S, Tsunezuka Y, Yamaguchi O, Okada M, Yoshimori K, Nakachi I, Gemma A, Azuma K, Kurimoto F, Tsubata Y, Fujita Y, Nagashima H, Asai G, Watanabe S, Miyazaki M, Hagiwara K, Nukiwa T, Morita S, Kobayashi K and Maemondo M 2019 Erlotinib plus bevacizumab versus erlotinib alone in patients with EGFR-positive advanced non-squamous non-small-cell lung cancer (NEJ026): interim analysis of an open-label, randomised, multicentre, phase 3 trial The Lancet Oncology 20 625-35

27. Go R S and Adjei A A 1999 Review of the comparative pharmacology and clinical activity of cisplatin and carboplatin J Clin Oncol 17 409-22

28. Wagstaff A J, Ward A, Benfield P and Heel R C 1989 Carboplatin. A preliminary review of its pharmacodynamic and pharmacokinetic properties and therapeutic efficacy in the treatment of cancer Drugs 37 162-90

29. Pinto A L and Lippard S J 1985 Binding of the antitumor drug cis-diamminedichloroplatinum(II) (cisplatin) to DNA Biochimica et biophysica acta $\mathbf{7 8 0}$ 167-80

30. Manohar S and Leung N 2018 Cisplatin nephrotoxicity: a review of the literature Journal of nephrology 31 15-25

31. Plooy A C, van Dijk M and Lohman P H 1984 Induction and repair of DNA cross-links in chinese hamster ovary cells treated with various platinum coordination compounds in relation to platinum binding to DNA, cytotoxicity, mutagenicity, and antitumor activity Cancer Res 44 2043-51

32. Zwelling L A, Michaels S, Schwartz H, Dobson P P and Kohn K W 1981 DNA cross-linking as an indicator of sensitivity and resistance of mouse L1210 leukemia to cis-diamminedichloroplatinum(II) and Lphenylalanine mustard Cancer Res 41 640-9

33. Hongo A, Seki S, Akiyama K and Kudo T 1994 A comparison of in vitro platinum-DNA adduct formation between carboplatin and cisplatin The International journal of biochemistry 26 1009-16

34. Sharma S, Ganesh T, Kingston D G and Bane S 2007 Promotion of tubulin assembly by poorly soluble taxol analogs Analytical biochemistry 360 56-62

35. Karimi M, Bahrami S, Ravari S B, Zangabad P S, Mirshekari H, Bozorgomid M, Shahreza S, Sori M and Hamblin M R 2016 Albumin nanostructures as advanced drug delivery systems Expert opinion on drug delivery 13 1609-23

36. Desai N, Trieu V, Yao Z, Louie L, Ci S, Yang A, Tao C, De T, Beals B, Dykes D, Noker P, Yao R, Labao E, Hawkins M and Soon-Shiong P 2006 Increased antitumor activity, intratumor paclitaxel concentrations, and endothelial cell transport of cremophor-free, albumin-bound paclitaxel, ABI-007, compared with cremophor-based paclitaxel Clinical cancer research : an official journal of the American Association for Cancer Research 12 1317-24

37. John T A, Vogel S M, Tiruppathi C, Malik A B and Minshall R D 2003 Quantitative analysis of albumin uptake and transport in the rat microvessel endothelial monolayer American journal of physiology. Lung cellular and molecular physiology 284 L187-96

38. Carver L A and Schnitzer J E 2003 Caveolae: mining little caves for new cancer targets Nat Rev Cancer $\mathbf{3}$ 571-81

39. Simionescu M, Gafencu A and Antohe F 2002 Transcytosis of plasma macromolecules in endothelial cells: a cell biological survey Microscopy research and technique 57 269-88

40. Minshall R D, Tiruppathi C, Vogel S M and Malik A B 2002 Vesicle formation and trafficking in endothelial cells and regulation of endothelial barrier function Histochemistry and cell biology 117 105-12

41. Vogel S M, Minshall R D, Pilipović M, Tiruppathi C and Malik A B 2001 Albumin uptake and transcytosis in endothelial cells in vivo induced by albuminbinding protein American journal of physiology. Lung cellular and molecular physiology 281 L1512-22

42. Schnitzer J E and Oh P 1994 Albondin-mediated capillary permeability to albumin. Differential role of receptors in endothelial transcytosis and endocytosis of native and modified albumins The Journal of biological chemistry $2696072-82$

43. Nyman D W, Campbell K J, Hersh E, Long K, Richardson K, Trieu V, Desai N, Hawkins M J and Von Hoff D D 2005 Phase I and pharmacokinetics trial of ABI-007, a novel nanoparticle formulation of 
paclitaxel in patients with advanced nonhematologic malignancies J Clin Oncol 23 7785-93

44. Schilling U, Friedrich E A, Sinn H, Schrenk H H, Clorius J H and Maier-Borst W 1992 Design of compounds having enhanced tumour uptake, using serum albumin as a carrier--Part II. In vivo studies International journal of radiation applications and instrumentation. Part B, Nuclear medicine and biology 19 685-95

45. Sage H 1986 Culture shock. Selective uptake and rapid release of a novel serum protein by endothelial cells in vitro The Journal of biological chemistry $\mathbf{2 6 1}$ 7082-92

46. Sage H, Johnson $C$ and Bornstein P 1984 Characterization of a novel serum albumin-binding glycoprotein secreted by endothelial cells in culture The Journal of biological chemistry 259 3993-4007

47. Gradishar W J 2006 Albumin-bound paclitaxel: a next-generation taxane Expert opinion on pharmacotherapy 7 1041-53

48. Korman A J, Peggs K S and Allison J P 2006 Checkpoint blockade in cancer immunotherapy Advances in immunology 90 297-339

49. Zang X and Allison J P 2007 The B7 family and cancer therapy: costimulation and coinhibition Clinical cancer research : an official journal of the American Association for Cancer Research 13 52719

50. Krummel M F and Allison J P 1995 CD28 and CTLA4 have opposing effects on the response of T cells to stimulation The Journal of experimental medicine 182 459-65

51. Patsoukis N, Wang Q, Strauss L, Boussiotis V A, Patsoukis N, Wang Q, Strauss L and Boussiotis V A 2020 Revisiting the PD-1 pathway Science Advances 6

52. Ohaegbulam K C, Assal A, Lazar-Molnar E, Yao Y and Zang X 2015 Human cancer immunotherapy with antibodies to the PD-1 and PD-L1 pathway Trends in molecular medicine 21 24-33

53. Dong H, Strome S E, Salomao D R, Tamura H, Hirano F, Flies D B, Roche P C, Lu J, Zhu G, Tamada K, Lennon V A, Celis E and Chen L 2002 Tumorassociated B7-H1 promotes T-cell apoptosis: a potential mechanism of immune evasion Nature medicine 8 793-800

54. Aydin A M, Woldu S L, Hutchinson R C, Boegemann M, Bagrodia A, Lotan Y, Margulis V and Krabbe L M 2017 Spotlight on atezolizumab and its potential in the treatment of advanced urothelial bladder cancer Onco Targets Ther 10 1487-502

55. Hellmann M D, Paz-Ares L, Bernabe Caro R, Zurawski B, Kim S W, Carcereny Costa E, Park K, Alexandru A, Lupinacci L, de la Mora Jimenez E, Sakai H, Albert I, Vergnenegre A, Peters S, Syrigos K, Barlesi F, Reck M, Borghaei H, Brahmer J R, O'Byrne K J, Geese W J, Bhagavatheeswaran P, Rabindran S K, Kasinathan R S, Nathan F E and Ramalingam S S 2019 Nivolumab plus Ipilimumab in
Advanced Non-Small-Cell Lung Cancer $N$ Engl $J$ Med 381 2020-31

56. West H, McCleod M, Hussein M, Morabito A, Rittmeyer A, Conter H J, Kopp H-G, Daniel D, McCune S, Mekhail T, Zer A, Reinmuth N, Sadiq A, Sandler A, Lin W, Ochi Lohmann T, Archer V, Wang L, Kowanetz M and Cappuzzo F 2019 Atezolizumab in combination with carboplatin plus nab-paclitaxel chemotherapy compared with chemotherapy alone as first-line treatment for metastatic non-squamous nonsmall-cell lung cancer (IMpower130): a multicentre, randomised, open-label, phase 3 trial The Lancet Oncology 20 924-37 